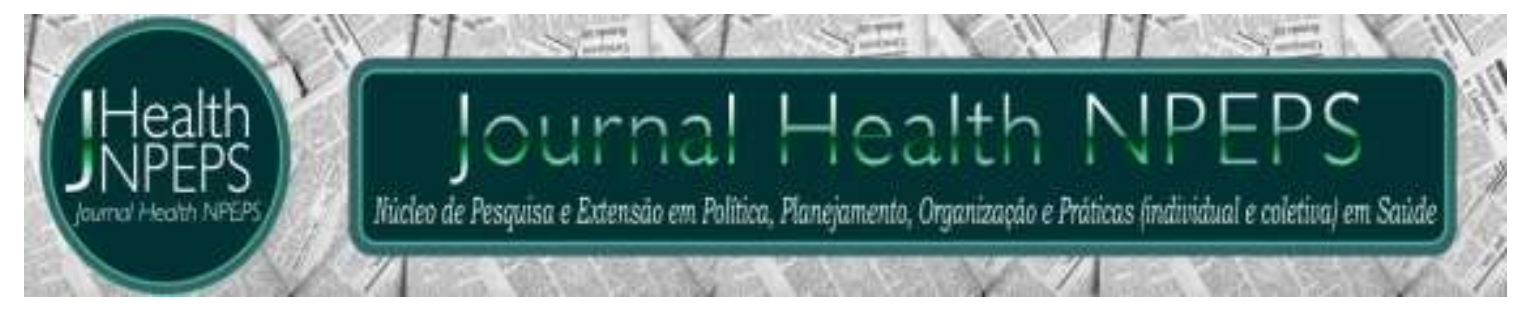

http://dx.doi.org/10.30681/252610103327

ENSAIO TEÓRICO-REFLEXIVO

\title{
Reflexão sobre a classificação de risco como tendência para o pronto-socorro infantil
}

\section{Reflection on risk classification as a trend for the infant emergency room \\ La reflexión sobre la calificación de riesgo como una tendencia para la emergencia del niño}

\section{Renato Rodrigues Rosa ${ }^{1}$, Karynne Borges Cabral${ }^{2}$, Cristiane Chagas Teixeira $^{3}$, Fernando Duarte Cabral ${ }^{4}$}

\section{RESUMO}

Objetivo: explanar acerca da classificação de risco como uma tendência para o serviço de pronto-socorro infantil. Método: trata-se de um ensaio teórico-reflexivo, realizado no período de maio de 2017 a outubro de 2018, fundamentando na literatura encontrada nas bases de dados: PubMed, CINAHL e LILACS. Os descritores utilizados foram: "Medição de Risco", "Serviços Médicos de Emergência", "Serviço Hospitalar de Emergência", "Pediatria”, “Identificação da Emergência”; "Triagem” e "Superlotação". Foram utilizados como critérios de inclusão: publicações em português, inglês e espanhol, no período de 2000 a 2016, com textos completos disponíveis gratuitamente. Excluídas as publicações que não se relacionavam à temática do estudo e que apresentassem duplicidade. Resultados: a classificação de risco mostra-se como tendência, com possibilidade de avançar, uma vez que a solução de muitos dos fatores que contribuem para a superlotação dos prontossocorros acarretaria maiores custos financeiros do que a implantação da classificação de risco infantil. Considerações Finais: a classificação de risco pode contribuir para a organização dos atendimentos ao cliente pediátrico que, procura por atendimento em prontos-socorros sem critérios clínicos.

\footnotetext{
${ }^{1}$ Médico e Administrador. Sargento do Corpo de Bombeiros Militar do Estado de Goiás. Goiânia, Goiás, Brasil. Email: renatomedr3@outlook.com ORCID ID: https://orcid.org/0000-0002-6646-3847 Autor principal Endereço para correspondência: Avenida C-206, esquina com Avenida C-198, Jardim América - Goiânia-GO. CEP 74270-060.

${ }^{2}$ Enfermeira. Doutora em Enfermagem. Universidade Federal de Goiás (UFG). Goiânia, Goiás, Brasil. E-mail: karynneenf26@hotmail.com ORCID ID: https://orcid.org/0000-0002-5025-2746

${ }^{3}$ Enfermeira. Doutoranda em Enfermagem. Universidade Federal de Goiás (UFG). Goiânia - Goiás, Brasil. E-mail: cc-teixeira@hotmail.com ORCID ID: https://orcid.org/0000-0002-4752-0439

${ }^{4}$ Fisioterapeuta. Especialista em Unidade de Terapia Intensiva. Docente da Faculdade Objetivo de Rio Verde GO. Goiânia, Goiás, Brasil. E-mail: fernandofisio2@hotmail.com ORCID ID: https://orcid.org/0000-0001-5167$\underline{5858}$

Este artigo está licenciado sob forma de uma licença Creative Commons Atribuição 4.0 Internacional, que permite uso irrestrito, distribuição e reprodução em qualquer meio, desde que a publicação original seja corretamente citada.
} 
Descritores: Medição de Risco; Serviços Médicos de Emergência; Pediatria.

\section{ABSTRACT}

Objective: to explain about the classification of risk as a tendency for the emergency room service for children. Method: it is a theoretical-reflective essay, carried out from May 2017 to October 2018, based on the literature found in the databases: PubMed, CINAHL and LILACS. The descriptors used were: "Risk Measurement", "Emergency Medical Services", "Emergency Hospital Service", "Pediatrics", "Emergency Identification", "Screening" and "Overcrowding". Inclusion criteria were: publications in Portuguese, English and Spanish, from 2000 to 2016, with full texts available free of charge. Publications that were not related to the subject of the study and which presented duplicity were excluded. Results: the risk classification is a trend with the possibility of advancing, since the solution of many of the factors that contribute to the overcrowding of the emergency rooms would entail higher financial costs than the implantation of the classification of child risk. Final Considerations: risk classification may contribute to the organization of pediatric care to the patient that seeks care in emergency rooms without clinical criteria.

Descriptors: Risk Assessment; Emergency Medical Services; Pediatrics.

\section{RESUMEN}

Objetivo: explicar acerca de la clasificación de riesgo como una tendencia al servicio de socorro infantil. Método: se trata de un ensayo teórico-reflexivo, realizado en el período de mayo de 2017 a octubre de 2018, fundamentando en la literatura encontrada en las bases de datos: PubMed, CINAHL y LILACS. Los descriptores utilizados fueron: "Medición de Riesgo", "Servicios Médicos de Emergencia", "Servicio Hospitalario de Emergencia", "Pediatría", "Identificación de la Emergencia"; "Triage" y "Superlotación". Fueron utilizados como criterios de inclusión: publicaciones en portugués, Inglés y Español, de 2000 a 2016, con el texto completo de libre acceso. Excluidas las publicaciones que no se relacionaban a la temática del estudio y que presentasen duplicidad. Resultados: la clasificación de riesgo se muestra como tendencia, con posibilidad de avanzar, ya que la solución de muchos de los factores que contribuyen a la superpoblación de los prontos de socorro acarrearía mayores costos financieros que la implantación de la clasificación de riesgo infantil. Consideraciones finales: la clasificación de riesgo puede contribuir a la organización de las atenciones al cliente pediátrico que, busca por atención en prontos de socorro sin criterios clínicos.

Descriptores: Medición de Riesgo; Servicios Médicos de Urgencia; Pediatría.

\section{INTRODUÇÃO}

Os serviços de pronto-socorro têm como principal finalidade acolher e atender os usuários, por meio de uma rápida avaliação do quadro clínico e admissão imediata no sistema hospitalar. São caracterizados pelo atendimento nas 24 horas do dia, pela organização do serviço baseada na procura dos usuários e pelo foco no atendimento imediato ${ }^{1,2}$. De maneira semelhante a outros países do mundo, o Brasil apresenta no contexto desses 
serviços, as características de longas filas de espera para atendimento, excesso de usuários, estrutura física e recursos humanos deficientes ${ }^{3}$.

Diante disso, o Ministério da Saúde (MS) do Brasil, propôs a estratégia de avaliação e classificação de risco (AACR), como norteadora do atendimento dos serviços da rede de urgência e emergência, com vistas a solucionar os problemas oriundos da procura por esses serviços sem qualquer critério clínico ${ }^{4}$.

0 AACR trata-se de um método de seleção dos pacientes, com a finalidade de direcioná-lo para o atendimento, conforme a prioridade requerida pelo seu quadro clínico, de forma humanizada, com informação do seu tempo de espera, organizando o fluxo de pacientes no sistema e selecionando os meios mais adequados para o diagnóstico e tratamento, para o problema de saúde apresentado ${ }^{5}$, porém, tal estratégia parece ser mais difundida, apenas quando se refere ao atendimento de pacientes adultos.

Experiências com o público adulto nos serviços de urgência e emergência têm evidenciado a eficiência da estratégia de AACR para - atendimento aos pacientes considerados graves, diminuindo 0 tempo de espera e, por conseguinte as mortes evitáveis, antes comuns nas filas de atendimentos dos serviços públicos de saúde ${ }^{6-8}$.

A busca indiscriminada pelo atendimento em pronto-socorro por clientes pediátricos, aparentemente comum em nosso país, também é evidenciada no cenário internacional. Em estudo que incluiu todos os pacientes com idade menor que 15 anos, realizado em 12 hospitais belgas, com o objetivo de avaliar a magnitude do fenômeno da procura pelo departamento de urgência, por casos não urgentes, mostrou que quase $40 \%$ de todos os atendimentos pediátricos, não exigiam atendimento emergencial ${ }^{9}$. Apesar disso, o uso da estratégia de classificação de risco no pronto-socorro infantil parece pouco difundida e estudada. Tal fato é evidenciado pelas raras publicações que abordam essa temática tanto a nível nacional quanto internacional.

Diante da problemática da superlotação do pronto-socorro pediátrico, agravada pela procura indiscriminada por esses serviços, que é por sua vez, sabidamente gerada pela oferta de serviços de saúde muito aquém da procura por seus usuários, pretendeu-se realizar uma 
reflexão sobre a classificação de risco como uma tendência para o serviço de pronto-socorro infantil.

Para o desenvolvimento deste estudo, questiona-se: a classificação de risco é capaz de vislumbrar uma possível melhoria do atendimento nos serviços de pronto-socorro infantil? A utilização de estratégias voltadas para minimizar os problemas da superlotação, com a priorização do atendimento a criança grave, contribui para qualidade desse tipo de atendimento? Diante disso, o presente estudo teve como objetivo explanar acerca da classificação de risco como uma tendência para o serviço de pronto-socorro infantil.

\section{MÉTODO}

Trata-se de um ensaio teóricoreflexivo ${ }^{10}$, realizado no período de maio a outubro de 2017, fundamentado na literatura encontrada nas bases de dados: National Library of Medicine National Institutes of Health (PubMed), Cumulative Index to Nursing and Allied Health Literature (CINAHL) e Literatura Latino-Americana e do Caribe em Ciências da Saúde (LILACS). Os descritores controlados selecionados nos Descritores em Ciências da Saúde (DeCS) da Biblioteca Virtual em Saúde (BVS) foram: "Medição de Risco", "Serviços Médicos de Emergência", "Serviço Hospitalar de Emergência”, "Pediatria”, “Identificação da Emergência”; “Triagem”, e como descritor não-controlado (palavrachave): “Superlotação". Os termos foram combinados de diferentes formas para garantir busca ampla. Foram utilizados como critérios de inclusão: publicações em português, espanhol e inglês, no período de 2000 a 2016, com textos completos disponíveis gratuitamente. Foram excluídas as publicações que não se relacionavam à temática do estudo e que apresentassem duplicidade.

Para a extração dos dados, foi elaborado um instrumento composto de itens relativos à identificação das publicações,

características metodológicas e avaliação do rigor metodológico. A coleta de dados foi realizada por dois pesquisadores, de forma independente. Posteriormente, as seleções foram confrontadas, a fim de verificar a compatibilidade dos achados e discutir as divergências. Os dados foram discutidos descritivamente, o que resultou em 
duas categorias, sendo a primeira " $A$ superlotação do pronto-socorro infantil" e a segunda a "Classificação de risco".

\section{A superlotação do pronto-socorro infantil}

Ao longo do tempo, os sistemas que visam estratificar ou classificar o risco dos pacientes ganharam destaque no cenário mundial dos serviços de urgência e emergência. Entretanto, no Brasil tal estratégia ganhou força somente após a publicação da Política Nacional de Humanização (PNH) no ano de 2004, que determinou a obrigatoriedade da implantação da classificação de risco nos serviços de urgência e emergência, com o intuito de priorizar os atendimentos graves, organizar as filas de espera e evitar mortes por retardo no atendimento médico ${ }^{11,12}$.

A busca indiscriminada pelos serviços de urgência e emergência sem critérios clínicos, tanto no cenário nacional como internacional demonstra, a necessidade de buscar estratégias de direcionamento dos atendimentos prioritários para além do público adulto incluindo a criança como parte inerente desse processo, uma vez que, a busca pelo prontosocorro sem qualquer critério clínico parece comum também a essa clientela.

No entanto, sabe-se que o uso inadequado do pronto-socorro por pacientes pediátricos é predominantemente associado a fatores organizacionais e culturais. Assim, o acesso, a equidade, a qualidade do atendimento, recursos humanos e médicos disponíveis são fatores que precisam ser levados em conta para projetar um modelo financeiramente sustentável de cuidados para os doentes pediátricos 9 .

A elevada magnitude de crianças com problemas de saúde que poderiam ser atendidas na atenção básica é preocupante, uma vez que, traz repercussão negativa sobre a qualidade da assistência prestada no pronto-socorro, tanto para as crianças que realmente necessitam dos serviços de urgências, como para as que precisam da atenção a saúde de nível ambulatorial, uma vez que, o atendimento prestado no prontosocorro visa resolver a queixa do paciente e este, muitas vezes, não é encaminhado para a Unidade Básica, o que pode comprometer a 
integralidade da saúde e do serviço ofertado ${ }^{13}$.

No entanto, a busca pelo atendimento no pronto-socorro por mães de crianças que poderiam ter suas queixas solucionadas na rede básica de saúde muitas vezes, justifica-se pela qualidade do atendimento, pela acessibilidade e em alguns casos pela ausência do médico nas Unidades Básicas de Saúde ${ }^{13}$. Assim, o costume de buscar hospitais, pronto-socorro e pronto atendimento, como primeira opção para o atendimento não urgente, sobrecarrega essas unidades e os profissionais de saúde, além de gerar preocupação para os gestores dos sistemas de saúde ${ }^{14}$. Tal fato reforça a necessidade de buscar estratégias que visem sanar ou ao menos minimizar a problemática da superlotação desses serviços.

\section{Classificação de risco}

A prática da classificação de risco de pacientes adultos é comum e facilmente encontrada, tantos nos serviços de saúde nacionais como nos internacionais. Diversos protocolos foram estruturados e validados e estão em uso sólido em muitos países.
Dentre os protocolos de classificação de risco mais conhecidos estão o Australalian Triage Scale (ATS) $)^{15}$ da Austrália, Canadian Emergency Department Triageand Acuity Scale $(\mathrm{CTAS})^{16}$ do Canadá, Manchester Triage System (MTS) ${ }^{17}$ do Reino Unido, Emergency Severity Index (ESI) ${ }^{18}$ dos Estados Unidos e Model Andorra de Triatje (MAT) ${ }^{19}$ da Espanha.

Em estudo anterior evidenciou-se que, a classificação de risco do paciente adulto é tida como importante fator que contribui para a melhor qualidade dos serviços de urgência e emergência, sobretudo por priorizar os atendimentos aos pacientes graves em detrimento dos menos graves, além de dar a oportunidade de criar vínculo de confiança entre os profissionais de saúde e os usuários do serviço8.

Mesmo diante de estudos que demonstram a eficiência da estratégia de classificação de risco nos serviços de urgência e emergência de pacientes adultos, tanto no Brasil como no mundo, essa prática direcionada ao pronto-socorro infantil, parece pouco estudada e ainda não consolidada.

A dificuldade de avaliar a criança quanto ao grau de sofrimento 
e risco de morte, talvez seja a explicação mais simples para a pouca difusão da estratégia de classificação de risco voltada para esse público. A análise subjetiva de crianças menores, aliada à falsa ideia de que "criança não pode esperar”, dificulta 0 engajamento dessa estratégia no pronto-socorro infantil. Além disso, sabe-se que, a formação acadêmica do enfermeiro ainda deixa a desejar, quando se trata de avaliar a criança como um todo.

No entanto, é necessário pensar na classificação de risco como uma tendência para o pronto-socorro infantil, com possibilidade de avançar em grande proporção, visto que, muitos fatores que contribuem para a superlotação dos prontos-socorros tanto adultos como infantis, demandam estruturação de outros setores de saúde, o que certamente acarretaria maiores custos financeiros, quando comparada a implantação da classificação de risco infantil. Entretanto, sabe-se que a chave para o atendimento seguro e eficaz das crianças é uma equipe clínica treinada, que se sinta confortável e confiante para cuidar dessa população 20 .
Torna-se necessário avançar em estudos e intervenções que possam contribuir para a implantação da classificação de risco no prontosocorro infantil. Porém, sabe-se que o sucesso de tal estratégia já aplicada ao paciente adulto indica uma tendência de adesão também voltada para a criança. Reconhece-se que tal estratégia por si só, não será capaz de sanar todos os problemas do prontosocorro infantil, entretanto, a priorização do atendimento da criança mais grave, torna-se extremamente valoroso em um lugar onde a procura sobressai em muito a oferta de serviços de saúde infantilil-23.

A limitação desse estudo, relaciona-se ao número reduzido de publicações voltadas para o cliente pediátrico e disponibilizadas de forma aberta nos periódicos de âmbito internacional.

\section{CONSIDERAÇÕES FINAIS}

A classificação de risco do cliente pediátrico mostra-se como uma possível tendência de organização do pronto-socorro infantil, uma vez que, pode trazer importantes benefícios para a qualidade desses serviços, já 
evidenciados em experiências com pacientes adultos. Reforça a necessidade de buscar estratégias que visem sanar a problemática da superlotação dos serviços, hoje baseada na procura por atendimento em pronto-socorro sem critérios clínicos, muitas vezes justificados pela falta de acesso a outros serviços de menor complexidade.

Diante disso, entende-se que a assistência em saúde de qualidade só será alcançada quando formos capazes de atingir um atendimento sólido e de qualidade ao cliente pediátrico. Considera-se que, a classificação de risco que vem sendo destaque no atendimento ao paciente adulto, também pode contribuir para a organização dos atendimentos ao cliente pediátrico.

No entanto, ressalta-se que, apenas classificar o risco, ordenar filas e orientar a população a buscar atendimento na unidade básica, sem garantir o seu acesso a ela, não contribui para 0 atendimento humanizado e nem tão pouco de qualidade, portanto, faz-se necessário buscar a estruturação de toda a rede de saúde, além de investir em educação em saúde da população para que se possa vislumbrar um atendimento eficaz, eficiente e com qualidade nos prontos-socorros infantis.

\section{REFERÊNCIAS}

1. Domiciano V, Fonseca AS. Tempo médio para o atendimento do cliente em um departamento de emergência de um hospital privado. Nursing (São Paulo). 2008; 11(119):182-8.

2. Tomberg JO, Cantarelli KJ, Guanilo MEE, Pai DD. Acolhimento com avaliação de risco no pronto socorro: caracterização dos atendimentos. Cienc Cuid Saude. 2013; 12(1):80-7.

3. Silva LG, Matsuda LM. Um olhar para a qualidade no processo de atendimento em um serviço de urgência público. Cienc Cuid Saude. 2012; 11 (suplem):121-28.

4. Ministério da Saúde (BR). Política Nacional de Humanização da Atenção e Gestão do SUS. Acolhimento e classificação de risco nos serviços de urgência. Brasília: Secretaria de Atenção à Saúde. Brasília: Ministéio da Saúde; 2009.

5. Moura ACF, Lopes AMS, Portela ALF, Sousa ID, Sousa SNS. 
Implantação do acolhimento com classificação de risco: um relato de experiência. Rev Multip Saúde HSM. 2013; 1(2):72-82.

6. Bellucci Júnior JA, Matsuda LM. Implantação do acolhimento com classificação de risco em serviço hospitalar de emergência: atuação do enfermeiro. Cienc Cuid Saude. 2012; 11(2):396-401.

7. Nascimento ERPd, Hilsendeger BR, Neth C, Belaver GM, Bertoncello KCG. Acolhimento com classificação de risco: avaliação dos profissionais de enfermagem de um serviço de emergência. Rev Eletrônica Enferm. 2011; 13(4):597-03.

8. Barbosa MC, Prata-Barbosa A, Cunha AJ, Lopes CS. CLARIPED: um Novo Instrumento para Classificação de Risco em Emergências Pediátricas. Rev Paul Pediatr. 2016; 34.

9. Benahmed N, Laokri S, Zhang WH, Verhaeghe N, Trybou J, Cohen L, et al. Determinants of nonurgent use of the emergency department for pediatric patients in 12 hospitals in Belgium. Eur $\mathrm{J}$ Pediatr. 2012; 171(12):1829-37.
10. Meneghetti FK. $O$ que é um Ensaio-Teórico? RAC. 2011; $15(2): 320-32$.

11. Ministério da Saúde (BR). HumanizaSUS: acolhimento com avaliação e classificação de risco um paradigma ético-estético no fazer em saúde. Brasília: Ministério da Saúde. SecretariaExecutiva. Núcleo Técnico da Política Nacional de Humanização; 2004.

12. Ministério da Saúde (BR). Política Nacional de Humanização. Brasília-DF: Ministéio da Saúde; 2013. Available from: http://bvsms. saude.gov.br/bvs/p ublicacoes/politica_nacional_hum anizacao_pnh_folheto.pdf.

13. Lima LMB, Almeida NMGS. Procura da emergência pediátrica pelas mães: implicações para a superlotação. Saúde debate. 2013; 37(96):51-61.

14. Rati RMS, Goulart LMHF, Alvim CG, Mota JAC. “Criança não pode esperar": a busca de serviço de urgência e emergência por mães e suas crianças em condições não urgentes. Ciênc Saúde Colet. 2013; 18(12):3663-72.

15. Australian

Government.

Department of Health and Ageing. 
Emergency triage education kit 2007 [cited 2015 Jan 19]. Available from: http://www.health.gov.au/intern et/main/publishing.nsf/Content/3 87970CE723E2BD8CA257BF0001DC 49F/ \$File/Triage\%20Education $\% 20$ Kit.pdf.

16. Murray M, Bullard M, Grafstein E; CTAS Natioanal Working Group; CEDIS National Working Group. Revisions to the Canadian Emergency Department Triage and Acuity Scale implementation guidelines. CJEM. 2004; 6(6):4217.

17. Grupo de Triagem de Manchester. Triagem do serviço de urgência. $2^{a}$ ed. Manchester - Portugual: BMJ Publisching; 2002.

18. Farrohknia $N$, Castrén $M$, Ehrenberg A, Lind L, Oredsson S, Jonsson $\mathrm{H}$, et al. Emergency Department Triage Scales and Their Components: A Systematic Review of the Scientific Evidence. Scand J Trauma Resusc Emerg Med. 2011;19:42.
19. Benavente RAS. Sistema de triaje en urgencias generales. Universitad Internacional de Andalucía [Internet]. 2014 [cited 2015 Jan 19 ]. Available from:http://dspace.unia.es/bitstr eam/handle/10334/2764/0561_Se rrano.pdf?sequence=1.

20. Hohenhaus SM. Someone whatching over me: observations in pediatric triage. J Emerg Nurs. 2006; 32(5):398-03.

21. Cheema B, Stephen C, Westwood A. Paediatric triage in South Africa. S Afr J Child Health. 2013; $7(2): 43-45$.

22. Chang YC, Ng CJ, Wu CT, Chen LC, Chen JC, Hsu KH. Effectiveness of a five-level Paediatric Triage System: an analysis of resource utilisation in the emergency department in Taiwan. Emerg Med J. 2013; 30(9):735-9.

23. Baracat ECE. Protocolos de triagem e classificação de risco em emergência pediátrica. Rev Paul Pediatr. 2016; 34(3):249-50.

Conflito de interesses: Os autores declaram não haver conflito de interesses.

Participação dos autores:

- Concepção: Rosa RR, Cabral KB, Teixeira CC, Cabral FD.

- Desenvolvimento: Rosa RR, Cabral KB, Teixeira CC, Cabral FD.

- Redação e revisão: Rosa RR, Cabral KB, Teixeira CC, Cabral FD. 
Como citar este artigo: Rosa RR, Cabral KB, Teixeira CC, Cabral FD. Reflexão sobre a classificação de risco como tendência para o pronto-socorro infantil. J Health NPEPS. 2019; 4(1):330-340.

Submissão: 25/01/2019

Aceito: $17 / 05 / 2019$

Publicado: 01/06/2019 